\title{
Urology in the Time of Coronavirus: Reduced Access to Urgent and Emergent Urological Care during the Coronavirus Disease 2019 Outbreak in Italy
}

\author{
Angelo Porreca ${ }^{a}$ Michele Colicchia $^{a}$ Daniele D'Agostino ${ }^{a}$ Michele Amenta $^{b}$ Alfio Corsaro ${ }^{b}$ \\ Stefano Zaramella ${ }^{c}$ Luisa Zegna ${ }^{c}$ Fabrizio Gallo ${ }^{d}$ Maurizio Schenone ${ }^{d}$ Giorgio Bozzini $^{e}$ \\ Alberto Calori ${ }^{\mathrm{e}}$ Antonio L. Pastore ${ }^{\mathrm{f}}$ Yazan Al Salhi $^{\mathrm{f}}$ Carmine Sciorio $^{g}$ Lorenzo Spirito $^{g}$ \\ Virginia Varca ${ }^{\text {h }}$ Carlo Marenghi ${ }^{\text {h }}$ Francesco Greco ${ }^{i}$ Vincenzo M. Altieri ${ }^{i}$ Paolo Verze $^{j}$ \\ Ciro Barba ${ }^{j}$ Alessandro Antonellik ${ }^{k}$ Maria A. Cerruto ${ }^{k}$ Roberto Falabellal Silvana Di Bello ${ }^{m}$ \\ Costantino Leonardo ${ }^{n}$ Antonio Tufano ${ }^{n}$ Alessandro Volpe ${ }^{\circ}$ Paolo Umari $^{\circ}$ Paolo Parma $^{p}$
} Mattia Nidini $^{p}$ Giovannalberto Pini ${ }^{q}$ Marco Borghesi ${ }^{r}$ Carlo Terrone ${ }^{r}$ Giovanni E. Cacciamani ${ }^{s}$ Maria C. Sighinolfit ${ }^{\mathrm{t}}$ Gian Maria Busetto ${ }^{\mathrm{u}}$ Alexandra M. Wennberg ${ }^{v}$ Marinella Finocchiarow Mario Falsaperlaw ${ }^{w}$ Marco Oderda ${ }^{x}$ Carlo Ceruti ${ }^{x}$ Bernardo Rocco ${ }^{t}$ Riccardo Schiavina ${ }^{y}$ Lorenzo Bianchi ${ }^{y}$ Andrea Mari ${ }^{z}$ Fabrizio Di Maida ${ }^{z}$ Orietta Dalpiaz ${ }^{A}$ Antonio Celia ${ }^{B}$ Marco Pirozzi ${ }^{B}$ Pierluigi Bove ${ }^{C}$ Valerio lacovelli ${ }^{C}$ Angelo Cafarelli ${ }^{D}$ Luca Cindolo ${ }^{E}$ Giovanni Ferrari $^{F}$ Lorenzo Gatti ${ }^{F}$ Giacomo Pirola ${ }^{G}$ Filippo Annino ${ }^{G}$ Luigi Pucci ${ }^{H}$ Daniele Romagnoli ${ }^{\mathrm{a}} \quad$ Walter $_{\text {Artibani }}{ }^{\mathrm{a}} \quad$ Andrea Minervini $^{\mathrm{z}}$

${ }^{a}$ Department of Urology, Policlinico Abano Terme, Abano Terme, Italy; ${ }^{b}$ Department of Urology, Ospedale Civile di Portogruaro, Portogruaro, Italy; ' Department of Urology, Biella Hospital, Ponderano, Italy; ${ }^{\mathrm{d}}$ Department of Surgery, Division of Urology, San Paolo Hospital, Savona, Italy; ${ }^{~}$ Department of Urology, ASST Valle Olona, Busto Arsizio, Italy; fDepartment of Medico-Surgical Sciences and Biotechnologies, Sapienza University of Rome, Urology Unit, ICOT Hospital

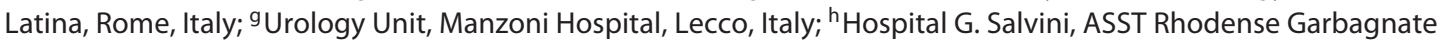
Milanese, Milan, Italy; 'Department of Urology, Humanitas Gavazzeni, Bergamo, Italy; jDepartment of Urology, Scuola Medica Salernitana, University of Salerno, Salerno, Italy; ${ }^{k}$ Department of Urology, Azienda Ospedaliera Universitaria Integrata of Verona, University of Verona, Verona, Italy; 'Department of Urology, Azienda Ospedaliera Regionale di Potenza, Potenza, Italy; ${ }^{\mathrm{m}}$ UO Pronto Soccorso, Accettazione, Medicina Urgenza, Azienda Ospedaliera Regionale di Potenza, Potenza, Italy; ${ }^{\mathrm{n}}$ Department of Urology, La Sapienza University of Rome, Rome, Italy; ${ }^{\circ}$ Division of Urology, Department of Translational Medicine, University of Eastern Piedmont, Maggiore della Carità Hospital, Novara, Italy;

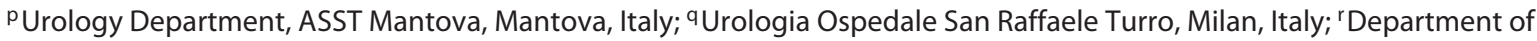
Urology, S. Martino Hospital, Department of Surgical and Diagnostic Sciences, University of Genoa, Genoa, Italy; ${ }^{s}$ USC Institute of Urology and Catherine and Joseph Aresty Department of Urology, Keck School of Medicine, University of Southern California, Los Angeles, CA, USA; ${ }^{t}$ Urology Department, University of Modena and Reggio Emilia, Modena, Italy; uDepartment of Urology, Sapienza Rome University Policlinico Umberto I, Rome, Italy; ${ }^{\vee}$ Department of Neurosciences

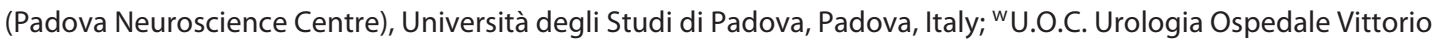
Emanuele, Catania, Italy; ${ }^{x}$ Università di Torino, Città della Salute e della Scienza di Torino, Turin, Italy; ' Department of Urology, University of Bologna, Bologna, Italy; ${ }^{z}$ Department of Urology, University of Florence, Florence, Italy; A Department of Urology, Medical University of Graz, Graz, Austria; ${ }^{B}$ Department of Urology, San Bassiano Hospital, Bassano del Grappa, Italy; CUrology Unit, San Carlo Di Nancy Hospital, GVM Care and Research, Rome, Italy; ${ }^{D}$ Department of Urology, Casa di Cura Villa Igea, Ancona, Italy; ${ }^{E}$ Department of Urology, Villa Stuart Casa di Cura, Rome, Italy; FDepartment of Urology, Hesperia Hospital, Modena, Italy; ${ }^{G}$ Department of Urology, San Donato Hospital, USL Toscana Sudest, Arezzo, Italy; ${ }^{\mathrm{H}}$ Department of Urology, AORN A. Cardarelli, Naples, Italy

Michele Colicchia

Department of Urology, Policlinico Abano Terme Piazza Cristoforo Colombo, 1 IT-35031 Abano Terme, PD (Italy) michelecolicchia@gmail.com 


\section{Keywords}

Coronavirus disease 2019 - Outbreak · Urological care · Italy

\section{Abstract}

Purpose: The coronavirus disease 2019 (COVID-19) pandemic has put a substantial burden on the Italian healthcare system, resulting in the restructuring of hospitals to care for $\mathrm{CO}$ VID-19 patients. However, this has likely impacted access to care for patients experiencing other conditions. We aimed to quantify the impact of COVID-19 on access to care for patients with urgent/emergent urological conditions throughout Italy. Materials and Methods: A questionnaire was sent to 33 urological units in the AGILE consortium, asking clinicians to report on the number of urgent/emergent urological patients seen and/or undergoing surgery over a 3-week period during the peak of the COVID-19 outbreak and a reference week prior to the outbreak. ANOVA and linear regression models were used to quantify these changes. Results: Data from 27 urological centres in Italy showed a decrease from 956 patients/week seen just prior to the outbreak to 291 patients/week seen by the end of the study period. There was a difference in the number of patients with urgent/emergent urological disease seen within/during the different weeks (all $p$ values $<0.05$ ). A significant decrease in the number of patients presenting with haematuria, urinary retention, urinary tract infection, scrotal pain, renal colic, or trauma and urgent/emergent cases that required surgery was reported (all $p$ values $<0.05$ ). Conclusion: In Italy, during the COVID-19 outbreak there has been a decrease in patients seeking help for urgent/emergent urological conditions. Restructuring of hospitals and clinics is mandatory to cope with the COVID-19 pandemic; however, the healthcare system should continue to provide adequate levels of care also to patients with other conditions.

(c) 2020 S. Karger AG, Basel

\section{Introduction}

At the end of December 2019, the World Health Organization reported on an outbreak of cases of pneumonia of an unknown aetiology in Wuhan City, Hubei Province, China. By January 7, 2020 the number of cases had grown rapidly, and the virus had been isolated and named SARS$\mathrm{CoV}-2$ - colloquially known as the coronavirus - and the resulting disease was named coronavirus disease 2019 (COVID-19) [1]. By the end of January 2020, the virus had spread beyond China, to 19 other countries. As of January 31, 2020, there were two confirmed cases in Italy
[2]. The outbreak in Italy started in the Lombardy region, but quickly spread to the whole country. By March 30, $2020,97,689$ confirmed cases and 10,779 deaths from the disease were reported in Italy [3].

In Italy, the exponential increase of cases led to an overtaxed health system, with a shortage of intensive care unit (ICU) beds and respiratory disease-related equipment. In response, hospitals and clinics were reorganized to respond to the growing outbreak. Internal medicine departments and ICUs shifted to focus on critically ill patients with COVID-19 [4]. This led to a disruption in care of patients presenting with other symptoms or emergent conditions, including urgent and emergent urological conditions.

Urological emergencies, including lower and upper urinary tract obstruction, obstructive pyelonephritis, gross haematuria with clot retention, and trauma, are not uncommon disorders. A delay to promptly recognize these emergencies may potentially lead to serious impairment such as renal failure, organ damage, or loss of sexual function [5-7].

According to World Bank Staff estimates, Italy has the second largest proportion of older adults in the world [8]. Older adults are more susceptible and more strongly affected by COVID-19, and they are also at greater risk of developing emergent urological conditions and relative complications.

The current multicentre study aimed to quantify changes in urgent and emergent urological care in Italy in the midst of the COVID-19 pandemic.

\section{Materials and Methods}

The AGILE group (Italian Group for Advanced Laparoscopic and Robotic Urologic Surgery) is a multicentre consortium consisting of 33 private and public urological departments and clinics across Italy. Data from 27 of these centres were included in this study. As of March 29, 2020 a standardized questionnaire (Fig. 1) was e-mailed to each of the departments and a quick response was requested. The questionnaire asked urologists to record and submit data with regard to patients presenting with urgent and emergent urological conditions (e.g., haematuria, urinary retention, urinary tract infection, scrotal pain, renal colic, and trauma) presenting at their respective institutions, including the number of emergent/urgent urological cases and the number of urgent/emergent cases that required surgery (e.g., endourological procedure, transurethral resection of bladder tumour, urethrotomy, and testicular detorsion). The included conditions and surgical procedures were considered representative markers to evaluate the usual urgent/emergent urological access. Accessibility to the emergency department operating facilities were reported for the weeks of February 24, 2020, March 2, 2020, March 9, 2020, and - as a 
Please report the overall number of emergent/urgent urological cases that presented at your institution in the given week

Please report the number of these cases that underwent surgery

Please report the number of cases presenting in each of the below

categories:

- Haematuria

- Urinary retention

- Urinary tract infection

- Scrotal pain

- Renal colic

- Trauma

Please report on the accessibility to emergency operating facilities:

- How many urgent/emergent interventions do you usually perform in a full week?

- Endourology (URS, ureteral stenting)

- Transurethral resection of bladder tumour

- Urethrotomy

- Testicular detorsion

The reference week could be of the clinicians' choosing, but it had to be prior to the outbreak (prior to $2 / 22$ ) and be a regular, full week of work (i.e., no holidays)

Fig. 1. E-mail questionnaire sent to the participating urological departments and centers. URS, ureteroscopy.

Table 1. Urgent and emergent urological patients, mean (standard deviation), ANOVA

\begin{tabular}{|c|c|c|c|c|c|}
\hline & $\begin{array}{l}\text { Reference } \\
\text { week }\end{array}$ & $\begin{array}{l}\text { COVID-19 } \\
\text { week } 1\end{array}$ & $\begin{array}{l}\text { COVID-19 } \\
\text { week } 2\end{array}$ & $\begin{array}{l}\text { COVID-19 } \\
\text { week } 3\end{array}$ & $p$ value \\
\hline Emergent/urgent cases, total & $35.4(24.3)$ & $20.3(15.3)$ & $17.8(14.2)$ & $10.8(11.8)$ & $<0.001$ \\
\hline Emergent/urgent cases in surgery, total & $8.7(7.4)$ & $4.4(4.1)$ & $3.6(3.3)$ & $3.4(3.8)$ & $<0.001$ \\
\hline \multicolumn{6}{|l|}{ Cases by type } \\
\hline Haematuria & $7.1(5.7)$ & $4.3(3.5)$ & $3.4(2.8)$ & $2.2(2.3)$ & $<0.001$ \\
\hline Urinary retention & $6.3(5.7)$ & $3.8(3.6)$ & $3.3(3.2)$ & $1.9(2.4)$ & $<0.001$ \\
\hline Urinary tract infection & $4.4(3.5)$ & $2.2(2.3)$ & $2.2(2.4)$ & $1.1(1.7)$ & $<0.001$ \\
\hline Scrotal pain & $3.7(4.0)$ & $2.1(2.3)$ & $1.6(1.8)$ & $0.9(1.2)$ & $<0.001$ \\
\hline Renal colic & $10.9(6.9)$ & $7.1(6.2)$ & $6.3(5.4)$ & $3.9(4.8)$ & $<0.001$ \\
\hline Trauma & $0.7(0.8)$ & $0.4(0.8)$ & $0.4(0.6)$ & $0.2(0.6)$ & 0.158 \\
\hline \multicolumn{6}{|l|}{ Emergency operating facilities } \\
\hline Endourology (URS, ureteral stenting) & $5.0(4.2)$ & $2.9(2.4)$ & $2.2(1.9)$ & $2.2(2.4)$ & 0.002 \\
\hline Transurethral resection of bladder tumour & $2.3(3.0)$ & $0.9(1.9)$ & $0.9(1.6)$ & $0.8(1.3)$ & 0.023 \\
\hline Urethrotomy & $0.5(0.8)$ & $0.3(0.6)$ & $0.1(0.5)$ & $0.1(0.4)$ & 0.081 \\
\hline Testicular detorsion & $0.8(0.8)$ & $0.4(0.8)$ & $0.2(0.5)$ & $0.2(0.4)$ & 0.006 \\
\hline
\end{tabular}

Bold indicates statistical significance $(p<0.05)$. COVID-19, coronavirus disease 2019; URS, ureteroscopy.

reference - a standard week (e.g., a week without holidays, conference, or other impending duties for the medical staff) before February 22, 2020. Day clinicians reported data in real time, based on the scheduling in their hospital and in accordance with their respective ethics committees. Additionally, we coded the 27 centres by geographic area as (1) Lombardy - the epicentre of the outbreak (5 centres), (2) neighbouring regions of Lombardy (11 centres), and (3) other Italian regions (11 centres).

Urological Care in the Time of Coronavirus
In the analyses, we summarized urgent/emergent urological cases and stratified these data by type of case that presented. We used ANOVA to examine the change in the number of cases presenting across the 4 -week period. We additionally examined the linear trajectory of the number of urgent/emergent urological cases seen across Italy during this period. All statistical analyses were performed with Stata version 15 (StataCorp LLC, College Station, TX, USA). 


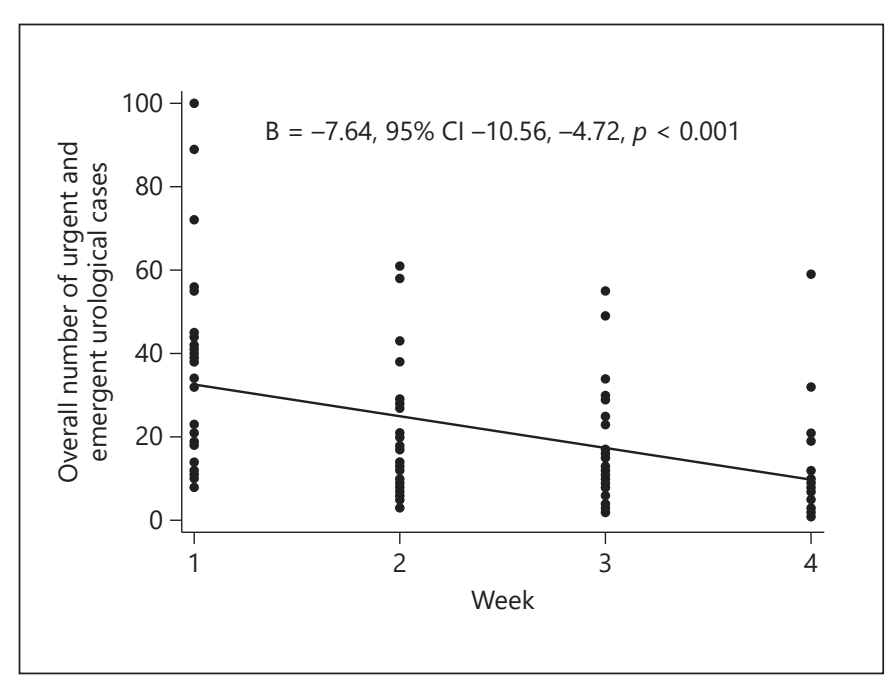

Fig. 2. Trend of overall urgent and emergent urological cases.

\section{Results}

Across 27 urological centres located throughout Italy, 956 patients with urgent/emergent condition were seen in the reference week (prior to the COVID-19 outbreak), and this number decreased to 291 by the week of March 9, 2020.

Using ANOVA models, we observed a statistically significant difference between weeks in number of overall $(p<0.001)$ and surgical $(p<0.001)$ urgent and emergent urological patients seen (Table 1 ). There were significant decreases in patients presenting with haematuria $(p<0.001)$, urinary retention $(p<0.001)$, urinary tract infection $(p<0.001)$, scrotal pain $(p<0.001)$, and renal colic $(p<0.001)$. Additionally, there were decreases in patients who underwent endourological procedures $(p=0.002)$, transurethral resection of bladder tumour $(p=0.023)$, and testicular detorsion $(p=0.006)$. The decrease was not significant for the number of trauma or urethrotomy patients. Examining trends across the study period, we observed a significant linear decrease in both overall $(\mathrm{B}=-7.64,95 \% \mathrm{CI}-10.56$ to $-4.72, p<0.001)$ and surgical $(\mathrm{B}=-1.66,95 \% \mathrm{CI}-2.51$ to $-0.81, p<0.001$ ) patients (Fig. 2,3 ). In sensitivity analyses, we investigated whether the area of Italy impacted the observed trends in decreases in patients seen, but found no evidence of interaction by geographic area. Finally, we additionally investigated whether there were any differences between private and public or academic and non-academic institutions, but found no differences.

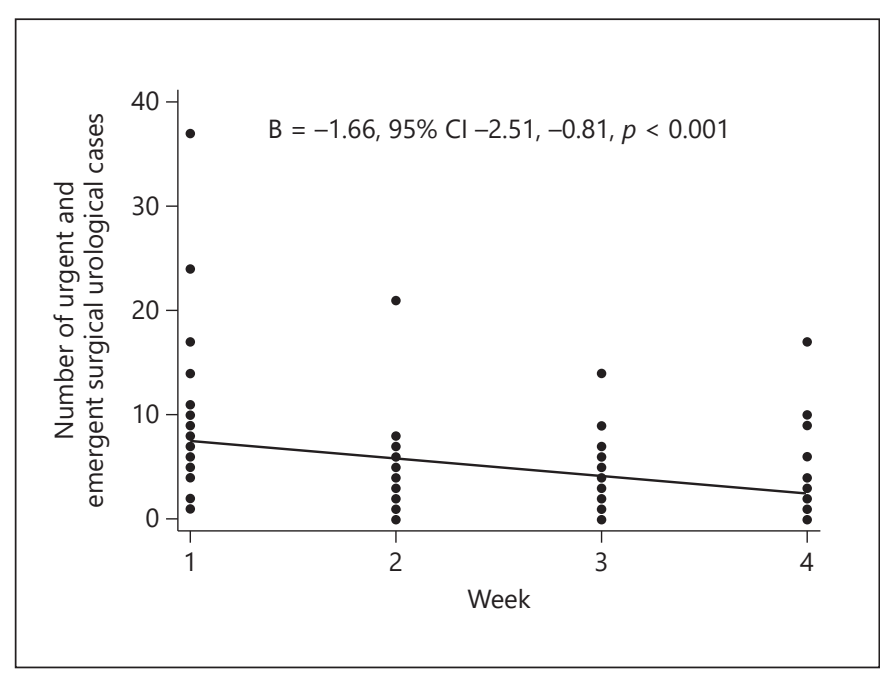

Fig. 3. Trend with fit line of urgent and emergent surgical urological cases.

\section{Discussion}

This multicentre study summarizes the change in access to urgent urological care in Italy during the COVID-19 pandemic. We found, across a 4-week period including one reference week and 3 weeks during the peak of the COVID-19 pandemic, a progressive and significant decline of the overall accesses of urgent urological cases and of cases requiring urgent surgical management. We observed a significant decrease in nearly all types of cases, including haematuria, urinary retention, urinary tract infection, scrotal pain, renal colic, endourological procedure, transurethral resection of bladder tumour, and testicular detorsion. Moreover, in linear regression models, there was a significant, linear decrease in both number of surgical cases and overall cases. Interestingly, there was no difference in the number of cases seen across the 4 -week period based on proximity to Lombardy, the epicentre of the outbreak.

This decreased number of accesses might be justified by the restrictions applied in Italy. The restriction in movements of the population except for necessity, work, and health circumstances (the so-called lockdown) in response to the growing pandemic might explain the reduction of patients who have hospital access for "not-essential" urgent urological consults. Also, staying home, resting, and avoiding exercise/outdoor activity may be a potential explanation for a decreased risk of certain conditions (e.g., urological trauma or testicular torsion) [9].

Overall, the COVID-19 pandemic has a significant impact on the management of the most important uro- 
logical diseases; the normal urological activity has been significantly reduced. For example, all this has led to relevant consequences on the execution of diagnostic tests even for the most frequent urological neoplasm including radiological examination [10]; consequently, access to even non-invasive treatments [11] and the consequent quality of life of this type of patients were affected [12].

The COVID-19 outbreak in Italy, and indeed throughout the world, illustrates the need for a different type of care during pandemics. The reorganization of healthcare facilities to care for the overwhelming number of severe patients with easily transmittable disease resulted in reduced care and resources for patients with other urgent and emergent conditions. This is apparent in the observed decrease in patients with urgent and emergent urological conditions reported here. The hospitalization rate for COVID-19 patients is approximately $50 \%$, while $16 \%$ of COVID-19 patients require ICU care [4]. This increased need to care for COVID-19 patients means there are fewer resources and personnel to care for patients with other urgent and emergent conditions. Indeed, healthcare workers are also at risk of becoming patients themselves. It has been estimated that $85 \%$ of healthcare workers will be exposed to the virus [13], and the International Council of Nurses estimated an infection rate of $9 \%$ in Italy [14].

This study was, as the situation warranted, conducted with careful haste. It provides a snapshot of the effect that the COVID-19 pandemic has on access to urgent and emergent care. However, although urological centres from across Italy were included and thus are likely representative of urological practice throughout the country, there could, of course, be aberrations, with only 27 centres reporting here. Additionally, although it is likely that these same patterns would be observed in any other specialty, this work is limited to urological data. Finally, although Italy's healthcare system is on par with those across Europe, some aspects of these findings may not be directly transferable to other European countries because of systematic and cultural differences.

Acknowledging these limitations, the current paper represents the first series so far exploring the impact of COVID-19 on the access to care for patients with urgent urological conditions. In particular, our findings strongly suggest that further protocols and facilities would be needed to provide care also for those patients who will inevitably develop other urgent conditions, irrespective of the COVID-19 pandemic. In this context, we identified future perspectives for the improvement of healthcare assistance: (1) create specific areas for the management and treatment of patients negative for COVID-19, either inside or outside of the existing structures; (2) improve education regarding infectious disease transmission for all healthcare providers; (3) meaningfully limit visitors' access to reduce the risk of contagion and, at the same time, to enhance patients' and employees' safety; and (4) delaying urological surgery should be considered whenever acceptable for the underlying disease, as reported by Stensland et al. [15], as a way of prioritizing the most urgent urological cases.

\section{Conclusions}

This multicentre study of urological units in Italy found a significant decrease in the number of patients treated for urgent and emergent urological conditions across a 3-week period during the COVID-19 pandemic. It is reasonable to assume that these patterns are being observed in other specialties as well. Italy has been substantially affected by the COVID-19 outbreak, with the second highest number of diseased individuals and the highest death rate of COVID-19 patients as of the end of March 2020 [3]. This outbreak has affected not only individuals with COVID-19, but also patients seeking care for other urgent and emergent conditions. Continued restructuring of the healthcare system is necessary to be able to continue providing a high level of care to all patients, even during times of pandemics.

\section{Statement of Ethics}

Proper consideration was given to any possible ethical issues. However, approval by an ethics commission was not applicable as this study is based on a survey.

\section{Disclosure Statement}

The authors have no conflicts of interest to declare.

\section{Funding Sources}

There are no funding sources. 


\section{Author Contributions}

Conception and design: Porreca, Colicchia, Minervini. Acquisition of data: Colicchia, Porreca, Amenta, Corsaro, Zaramella, Zegna, Gallo, Schenone, Bozzini, Calori, Pastore, Al Salhi, Sciorio, Spirito, Varca, Marenghi, Greco, Altieri, Verze, Barba, Antonelli, Cerruto, Falabella, Di Bello, Leonardo, Tufano, Volpe, Umari, Parma, Nidini, Borghesi, Terrone, Sighinolfi, Finocchiaro, Falsaperla,
Oderda, Ceruti, Rocco, Schiavina, Bianchi, Di Maida, Celia, Pirozzi, Bove, Iacovelli, Cindolo, Ferrari, Gatti, Pirola, Annino, Pucci, Romagnoli. Analysis and interpretation of data: Colicchia, Porreca, Wennberg. Drafting of the manuscript: Porreca, Colicchia. Critical revision of the manuscript for important intellectual content: Porreca, Colicchia, D’Agostino, Pini, Bozzini, Verze, Cacciamani, Busetto, Rocco, Mari, Dalpiaz, Cafarelli, Artibani. Statistical analysis: Colicchia, Wennberg. Supervision: Minervini.

\section{References}

1 World Health Organization. Novel Coronavirus (2019-nCoV): Situation Report - 12020. https://www.who.int/docs/default-source/ coronaviruse/situation-reports/20200121sitrep-1-2019-ncov.pdf?sfvrsn=20a99c10_4.

2 World Health Organization. Novel Coronavirus (2019-nCoV): Situation Report - 112020. www.who.int/docs/default-source/coronaviruse/situation-reports/20200131-sitrep11-ncov.pdf?sfvrsn=de7c0f7_4 [accessed March 30, 2020].

3 Johns Hopkins Coronavirus Resource Center. https://coronavirus.jhu.edu/map.html [accessed March 30, 2020].

4 Grasselli G, Pesenti A, Cecconi M. Critical Care Utilization for the COVID-19 Outbreak in Lombardy, Italy: Early Experience and Forecast During an Emergency Response. JAMA. 2020 Mar;323(16):1545.

5 Gratzke C, Bachmann A, Descazeaud A, Drake MJ, Madersbacher S, Mamoulakis C, et al. EAU Guidelines on the Assessment of Non-neurogenic Male Lower Urinary Tract Symptoms including Benign Prostatic Obstruction. Eur Urol. 2015 Jun;67(6):1099-109.
6 Türk C, Petř́k A, Sarica K, Seitz C, Skolarikos A, Straub M, et al. EAU Guidelines on Diagnosis and Conservative Management of Urolithiasis. Eur Urol. 2016 Mar;69(3):468-74.

7 Salonia A, Eardley I, Giuliano F, Hatzichristou D, Moncada I, Vardi Y, et al.; European Association of Urology. European Association of Urology guidelines on priapism. Eur Urol. 2014 Feb;65(2):480-9.

8 World Bank. Population ages 65 and above (\% of total population). World Dev Indic 2019.

9 DaJusta DG, Granberg CF, Villanueva C, Baker LA. Contemporary review of testicular torsion: new concepts, emerging technologies and potential therapeutics. J Pediatr Urol. 2013 Dec;9(6 6 Pt A):723-30.

10 Vagnoni V, Brunocilla E, Bianchi L, Porreca A, Borghesi M, Pultrone CV, et al. State of the art of PET/CT with 11-choline and 18F-fluorocholine in the diagnosis and follow-up of localized and locally advanced prostate cancer. Arch Esp Urol. 2015 Apr;68(3):354-70.

11 Grasso AA, Cozzi G, DE Lorenzis E, Ceruti C, Crivellaro S, Falsaperla M, et al. Multicenter analysis of pathological outcomes of patients eligible for active surveillance according to PRIAS criteria. Minerva Urol Nefrol. 2016 Jun;68(3):237-41.
12 Gacci M, Noale M, Artibani W, Bassi PF, Bertoni F, Bracarda S, et al.; Pros-IT CNR study group. Quality of Life After Prostate Cancer Diagnosis: data from the Pros-IT CNR. Eur Urol Focus. 2017 Oct;3(4-5):3214.

$13 \mathrm{Ng}$ K, Poon BH, Kiat Puar TH, Shan Quah JL, Loh WJ, Wong YJ, et al. COVID-19 and the risk to health care workers: a case report. Ann Intern Med. doi: 10.7326/L20-0175 [Epub ahead of print].

14 International Council of Nurses. ICN tells BBC World News viewers: Rising rate in COVID-19 infection amongst health workers requires urgent action. www.icn.ch/news/icntells-bbc-world-news-viewers-rising-rate-covid-19-infection-amongst-health-workers [accessed April 2, 2020].

15 Stensland KD, Morgan TM, Moinzadeh A, Lee CT, Briganti A, Catto J, et al. Considerations in the triage of urologic surgeries during the COVID-19 pandemic. Eur Urol. doi: 10.1016/j.eururo.2020.03.027 [Epub ahead of print]. 